TITLE:

\title{
Transmission of Lamb waves and resonance at an adhesive butt joint of plates
}

$\operatorname{AUTHOR}(\mathrm{S})$ :

Mori, Naoki; Biwa, Shiro

\section{CITATION:}

Mori, Naoki ... [et al]. Transmission of Lamb waves and resonance at an adhesive butt joint of plates. Ultrasonics 2016, 72: 80-88

ISSUE DATE:

2016-12

URL:

http://hdl.handle.net/2433/226611

\section{RIGHT:}

(c) 2016. This manuscript version is made available under the CC-BY-NC-ND 4.0 license

http://creativecommons.org/licenses/by-nc-nd/4.0/; The full-text file will be made open to the public on 01 December 2018 in accordance with publisher's 'Terms and Conditions for Self-Archiving'; この論文は出版社版でありません。引用 の際には出版社版をご確認ご利用ください。; This is not the published version. Please cite only the published version. 
Manuscript to appear in Ultrasonics

Transmission of Lamb waves and resonance at an adhesive butt joint of plates

\author{
Naoki Mori, Shiro Biwa*
}

Department of Aeronautics and Astronautics, Graduate School of Engineering, Kyoto University, Katsura, Nishikyo-ku, Kyoto 615-8540, Japan.

${ }^{*}$ Corresponding author. Tel.: +81-75-383-3796.

E-mail address: biwa@kuaero.kyoto-u.ac.jp (S. Biwa).

\begin{abstract}
The transmission behavior of Lamb waves and the possible occurrence of resonance at an adhesive butt joint of plates are studied experimentally. To this purpose, two $2.5-\mathrm{mm}$ thick aluminum alloy plates are bonded at their edges using cyanoacrylate-based adhesive. Bonded plate specimens with different joint conditions are prepared by changing the bonding procedure. The measurements are performed for the transmission characteristics of the lowest-order symmetric (S0) and antisymmetric (A0) Lamb modes for the frequency range of 0.4 to $0.6 \mathrm{MHz}$ below the cut-off frequency of the higher-order modes. The experimental results show that the transmission coefficients of the S0 and A0 modes exhibit different frequency-dependent characteristics depending on the joint condition. Furthermore, for the incidence of the S0 mode at the center frequency of $1 \mathrm{MHz}$, the transmitted S0 mode in weakly bonded specimens shows a long oscillation tail due to the resonance effect. The experimental results are discussed in the light of the theoretical results based on the spring-type interface model. The interfacial stiffnesses identified from the transmission coefficients are shown to be correlated with the bonding condition of the joint and give reasonable estimates of the resonance frequencies of weakly bonded specimens.
\end{abstract}

Keywords: Lamb wave, Adhesive joint, Transmission, Joint resonance, Interfacial stiffness 


\section{Introduction}

Guided elastic waves have received much attention in the area of nondestructive evaluation and structural health monitoring due to their capability of propagating relatively long distances along the structures. Lamb waves are known as guided waves propagating in a thin plate, and expected to offer an effective tool for the inspection of plate-like structures. Lamb waves exhibit complicated propagation behavior because of their dispersion and multi-modal properties. Therefore, numerous experimental and theoretical investigations have been reported [1-4] in order to obtain the precise understanding of Lamb waves interacting with various types of defects.

Lamb waves have also been applied to characterize the quality of bonding used in various plate structures [5-11]. Most of foregoing studies focused on lap joints or layered plates for which the plate surfaces are bonded by adhesives. For example, Lowe et al. [7] investigated the mode conversion behavior as well as the transmission characteristics of Lamb waves at an adhesively bonded lap joint. Heller et al. [8] used the dispersion relation of Lamb waves to characterize adhesive layers in plates. In contrast, there have been relatively few works reported for the Lamb wave interaction with butt-type joints of plates in spite of their simple geometry. This type of joints is less practical for adhesive bonding of plates, but offers basic insights regarding the behavior of Lamb waves. Predoi and Rousseau [10] studied the reflection and transmission of Lamb waves at a joint strip between elastic plates.

Recently, Mori et al. [12] analyzed the reflection and transmission characteristics of Lamb waves at a butt joint of plates based on the spring-type interface model, which has been frequently used to analyze the interaction of ultrasonic waves with various imperfect interfaces [13-22]. They demonstrated that the reflection and transmission coefficients of the lowest-order symmetric (S0) and antisymmetric (A0) Lamb modes at the joint vary with the frequency and the interfacial stiffnesses. Furthermore, Mori and Biwa [23] showed that the imperfect joint modeled as a spring-type interface exhibits resonance behavior which is analogous to the free-edge resonance of a plate. They found that the imperfect joint has two resonance frequencies which selectively depend on the normal and tangential stiffnesses. These studies are, however, limited to numerical analysis. Experimental verification of such findings is important from a fundamental point of view in order to elucidate the interaction of Lamb waves with imperfect joints. It can also give a background to the characterization of not only adhesive joints but also 
other imperfect interfaces in plate structures such as closed fatigue cracks [24-26] using Lamb waves.

The aim of the present study is to experimentally investigate the interaction of Lamb waves with an imperfect joint of plates. To this purpose, two aluminum alloy plates are bonded at their edges by adhesive to constitute an imperfect butt joint. Bonded plate specimens with different joint properties are prepared by applying different bonding procedures. The experiments are carried out to evaluate the transmission characteristics of the S0 and A0 Lamb modes and the possible resonance at the joint when subjected to the $\mathrm{S} 0$ mode incidence. The experimental results are discussed in the light of the theoretical results $[12,23]$ based on the spring-type interface model. In this paper, different modes of Lamb waves are denoted by S0, S1, S2, ... for symmetric modes and by A0, A1, A2, ... for antisymmetric modes according to conventional terminology [27].

\section{Experiment}

\subsection{Specimens}

Figure 1 shows the schematic of a plate specimen with an adhesive butt joint. Two square-shaped aluminum alloy plates (A5052, plate thickness $2.5 \mathrm{~mm}$ and side length $150 \mathrm{~mm}$ ) were bonded together at their edge faces (area $150 \times 2.5 \mathrm{~mm}^{2}$ ) using cyanoacrylate-based adhesive (Aron Alpha \#30114, Konishi Co., Ltd.). Four bonded plate specimens (Samples I-IV) were prepared with different bonding procedures. Namely, after the adhesives were applied on the edge faces, two plates were fixed to each other in stainless steel guide rails and subjected to different levels of compressive loading for different time durations. Table 1 shows the bonding conditions and the resulting adhesive thicknesses measured by an Olympus laser microscope (OLS1200). In Table 1, the nominal pressure is defined as the ratio of the applied compressive force to the area of the edge face $\left(150 \times 2.5 \mathrm{~mm}^{2}\right)$. These bonded specimens were kept in the guide rails to prevent flexure during two kinds of experiments described below. Furthermore, a single aluminum alloy plate (A5052, size $150 \mathrm{~mm} \times 300 \mathrm{~mm} \times 2.5 \mathrm{~mm}$ ) was employed as the reference plate specimen with no joint interface. This reference specimen was also kept in the guide rails to perform the measurement in the same configuration as that for the bonded specimens. 


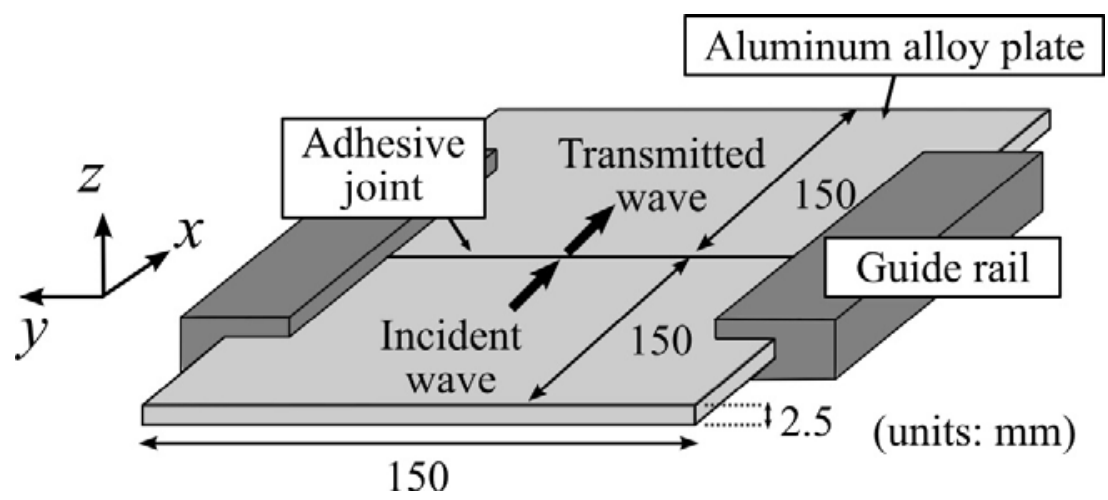

Fig. 1. Schematic of adhesively bonded aluminum alloy plates.

Table 1 Nominal pressure and compression time for the adhesively bonded specimens, and thicknesses of adhesive $h_{\mathrm{A}}$.

\begin{tabular}{c|ccc}
\hline \hline & Nominal pressure & Compression time & $h_{\mathrm{A}}[\mathrm{mm}]$ \\
\hline Sample I & $0.37 \mathrm{MPa}$ & $10 \mathrm{~min}$. & 0.11 \\
Sample II & $0.10 \mathrm{MPa}$ & $10 \mathrm{~min}$. & 0.13 \\
Sample III & $<0.03 \mathrm{MPa}$ & $10 \mathrm{~min}$. & 0.27 \\
Sample IV & $0.05 \mathrm{MPa}$ & $5 \mathrm{sec}$. & 0.16 \\
\hline \hline
\end{tabular}

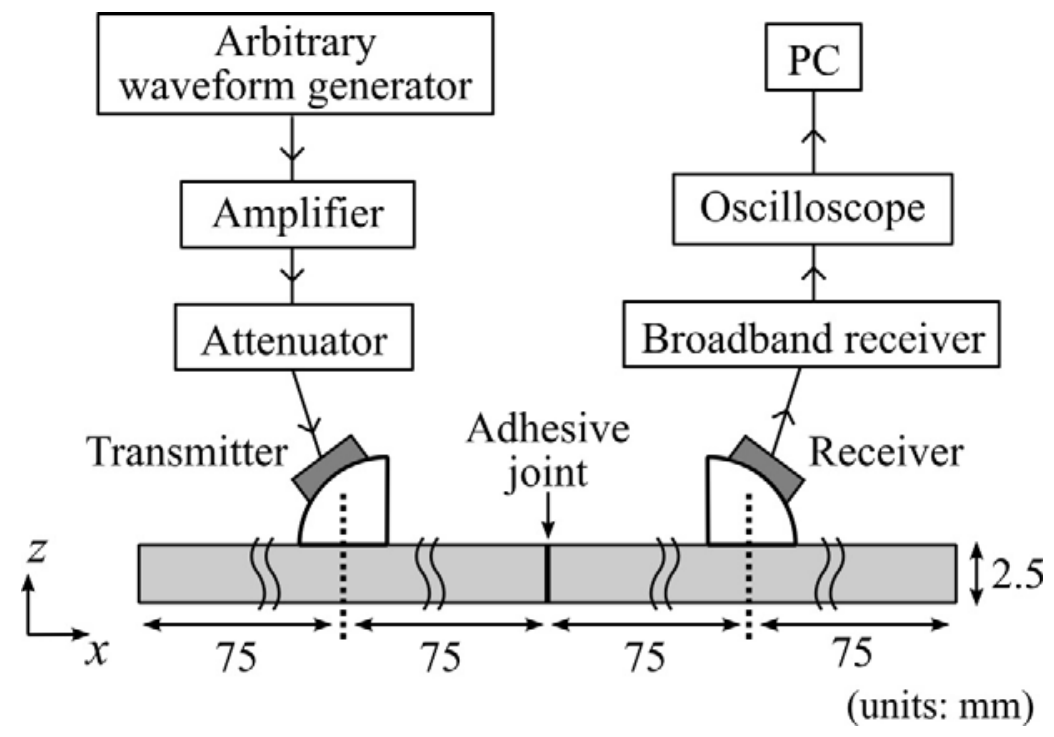

Fig. 2. Schematic of the experimental setup. 


\subsection{Experimental procedures}

\subsubsection{Transmission measurement of the lowest-order Lamb modes}

The transmission coefficients of the S0 and A0 Lamb modes were measured in a relatively low frequency range below the cut-off frequencies $(0.63 \mathrm{MHz}$ for the $2.5-\mathrm{mm}$ thick aluminum alloy plate) of higher-order Lamb modes. The setup for the measurement is schematically shown in Fig. 2. Two piezoelectric longitudinal wave transducers (Panametrics V413, nominal frequency $0.5 \mathrm{MHz}$ ) were fitted to adjustable-angle wedges and mounted on the surface of the specimen with liquid couplant. A Gaussian-modulated tone-burst signal given by

$$
g(t)=\exp \left[-\left(\frac{t-t_{0}}{\sigma_{0}}\right)^{2}\right] \sin \left[2 \pi f_{0}\left(t-t_{0}\right)\right],
$$

was generated by an Agilent arbitrary waveform generator (33220A), and sent to the emitting transducer via a Thamway amplifier (T145-7516B) and a RITEC attenuator (RA-30). In Eq. (1), $t$ is the time, $f_{0}=0.5 \mathrm{MHz}$ is the center frequency, and the other parameters are given as $t_{0}=50 \mu \mathrm{s}$ and $\sigma_{0}=3 \mu \mathrm{s}$.

(a)

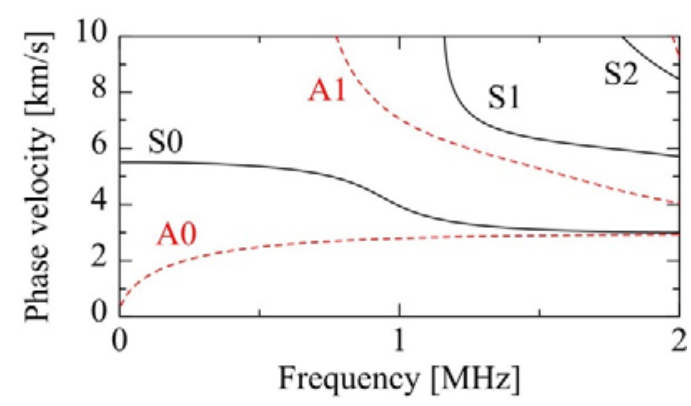

(b)

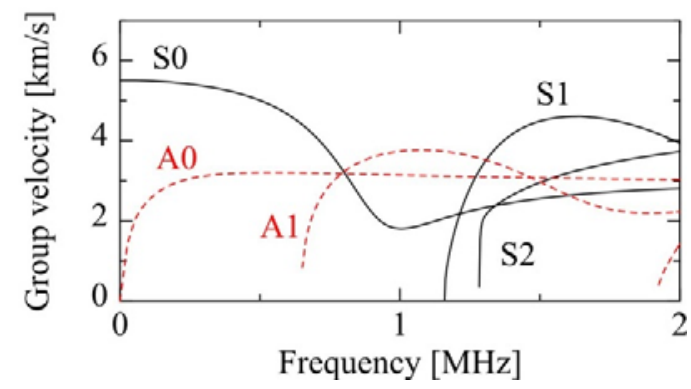

Fig. 3. (a) Phase velocities and (b) group velocities of different Lamb modes for the 2.5-mm thick aluminum plate (the velocities of longitudinal and transverse waves are $c_{\mathrm{L}}=6.4 \mathrm{~km} / \mathrm{s}$ and $c_{\mathrm{T}}=3.17 \mathrm{~km} / \mathrm{s}$, respectively). 
According to Snell's law, a particular Lamb mode can be excited and detected when the wedge angle $\theta$ is

$$
\theta=\sin ^{-1}\left(\frac{C_{\mathrm{w}}}{c}\right)
$$

where $c_{\mathrm{w}}=2.72 \mathrm{~km} / \mathrm{s}$ is the longitudinal wave velocity of the wedge material (PMMA) and $c$ is the phase velocity of the desired Lamb mode. The dispersion curves of Lamb modes for the aluminum plate are shown in Fig. 3. At $0.5 \mathrm{MHz}$, the S0 mode has the phase velocity $5.4 \mathrm{~km} / \mathrm{s}$ and Eq. (2) gives the wedge angle $\theta=31 \mathrm{deg}$. In the measurement, the wedge angle was set as 30 deg for the S0 mode incidence. On the other hand, the A0 mode has the phase velocity $2.5 \mathrm{~km} / \mathrm{s}$ at $0.5 \mathrm{MHz}$, for which there is no angle satisfying Eq. (2). As a consequence, a relatively large angle of $60 \mathrm{deg}$ was employed in the measurement and its validity to excite the A0 mode was checked experimentally (see Sec. 3.1).

The distance between the two wedges was fixed to $150 \mathrm{~mm}$. The transmitted wave measured by the receiving transducer was recorded as digital data with a KEYSIGHT digital oscilloscope (DSOS054A) and transferred to a PC after averaging over 64 synchronized signals. The measured waveform was then analyzed in the frequency domain by FFT (fast Fourier transform) to obtain the amplitude spectrum. The amplitude transmission coefficient was obtained at each frequency by dividing the spectral amplitude by the corresponding amplitude of the reference waveform measured for the reference plate in the same manner.

\subsubsection{Measurement of the resonance effect for the SO mode incidence}

As described in Ref. [23], when an imperfect joint of plates is modeled as a linear spring-type interface, the joint resonance occurs for the S0 mode incidence at two frequencies below the cut-off frequencies of higher-order symmetric Lamb modes $(1.27 \mathrm{MHz}$ for the $2.5-\mathrm{mm}$ thick aluminum alloy plate). The numerical simulation in the time domain [28] has shown that the resonance effect of the imperfect joint gives rise to long oscillation tails in the reflected and transmitted waveforms analogously to the free-edge resonance [29]. The present measurement aims to detect the long-time oscillation in the transmitted waveforms of the adhesively bonded specimens for the S0 mode incidence.

The experimental setup was the same as that for the measurement of the transmission coefficients shown in Fig. 2, except for the excitation frequency and the transducers. The input 
waveform was given by Eq. (1) but the parameters are $t_{0}=50 \mu \mathrm{s}$ and $\sigma_{0}=4 \mu \mathrm{s}$. The center frequency is $f_{0}=1 \mathrm{MHz}$. Two longitudinal wave transducers with the nominal frequency $1 \mathrm{MHz}$ (Panametrics V401) were utilized since the frequency range of interest was higher than that of the previous measurement. As shown in Fig. 3, the S0, A0, and A1 Lamb modes can propagate in the plate at the frequency of $1 \mathrm{MHz}$. Equation (2) predicts that the S0 mode (phase velocity 3.9 $\mathrm{km} / \mathrm{s}$ ) can be generated and detected dominantly at the wedge angle of $\theta=44 \mathrm{deg}$. In the measurement, the wedge angle was set as $\theta=45 \mathrm{deg}$. The waveforms of the S0 mode transmitted across the adhesive joint were compared to the waveform of the S0 mode which propagated the same distance in the reference plate.

\section{Experimental results}

\subsection{Transmission coefficients of the lowest-order Lamb modes}

The experimental results for the reference plate are first examined to confirm the generation and detection of Lamb waves with the two wedge transducers. The obtained waveforms are shown for the wedge angles $\theta=30 \mathrm{deg}$ and $\theta=60 \mathrm{deg}$ in Figs. 4 (a) and (b), respectively. The arrival times of the main wave packet are clearly different for $\theta=30 \mathrm{deg}$ and $\theta=60 \mathrm{deg}$ because different

(a)

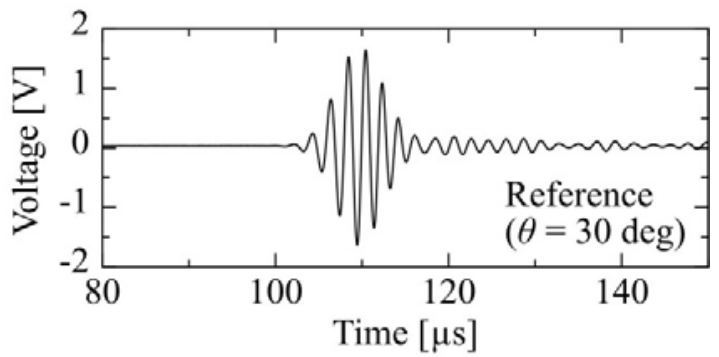

(b)

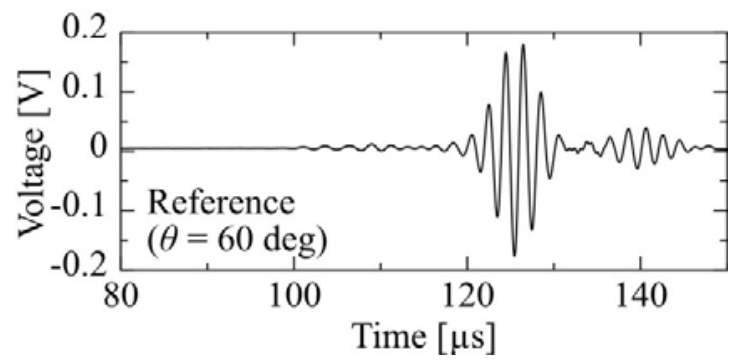

Fig. 4. Waveforms measured in the reference plate when (a) $\theta=30 \mathrm{deg}$ and (b) $\theta=60$ deg. The center frequency of the input waveform is $0.5 \mathrm{MHz}$. 
modes are excited and detected dominantly due to the difference of the wedge angle. At $0.5 \mathrm{MHz}$, the S0 and A0 modes are expected to arrive at $110 \mu \mathrm{s}$ and $127 \mu \mathrm{s}$, respectively, according to their group velocities $(5.0 \mathrm{~km} / \mathrm{s}$ and $3.2 \mathrm{~km} / \mathrm{s}$, respectively). Therefore the main wave packet in Fig. 4 (a) is judged to be the S0 mode, while the one in Fig. 4 (b) is the A0 mode. Weak tails following the main wave packet result from reflections at boundaries of the wedges, plate edges, etc.

Figures 5 (a)-(d) show the transmitted waves of the adhesively bonded specimens (Samples I-IV) when $\theta=30 \mathrm{deg}$. It is clearly observed that Sample IV exhibits particularly decreased transmitted amplitude. The wave packets from $100 \mu$ s to $120 \mu$ s corresponding to the S0

(a)

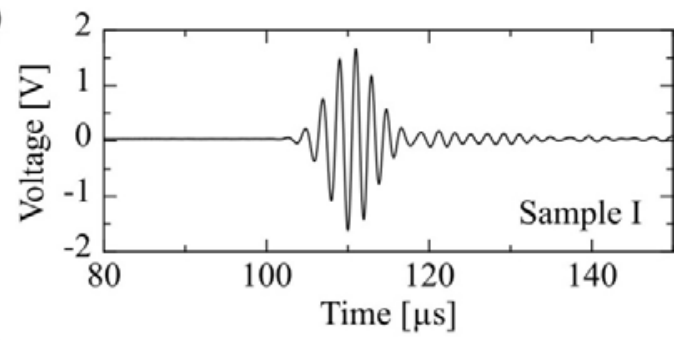

(b)

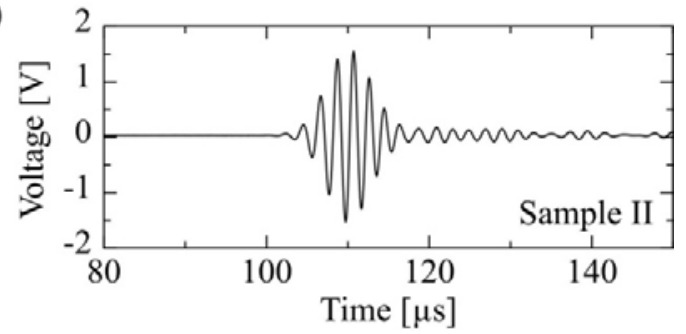

(c)

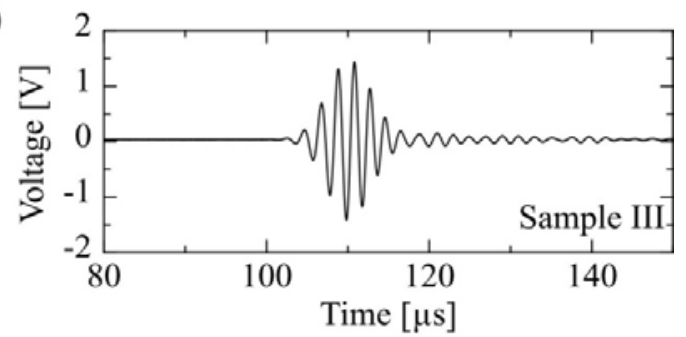

(d)

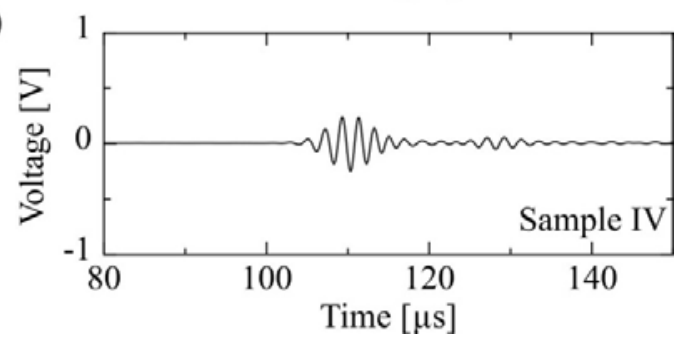

Fig. 5. Measured waveforms of Samples (a) I, (b) II, (c) III, and (d) IV for the S0 mode incidence $(\theta=30 \mathrm{deg})$. 
mode were analyzed by the FFT to calculate the amplitude spectra. The transmission coefficient of the $\mathrm{S} 0$ mode is obtained as a function of the frequency $f$ by

$$
T_{\mathrm{S} 0}(f)=\frac{B_{\mathrm{S} 0}(f)}{A_{\mathrm{S} 0}(f)}
$$

where $A_{\mathrm{S} 0}(f)$ and $B_{\mathrm{S} 0}(f)$ denote the $\mathrm{S} 0$ mode amplitudes for the reference and the bonded specimens, respectively. The S0 mode transmission coefficients for four bonded plate specimens are shown in Fig. 6. Samples II-IV show slightly decreasing behavior with the frequency, while Sample I exhibits almost perfect transmission.

Likewise, the transmitted waves were measured for the A0 mode incidence ( $\theta=60 \mathrm{deg})$, as shown in Figs. 7 (a)-(d). The maximum amplitudes of the A0 mode in Samples II-IV are smaller than the reference plate (Fig. 4 b) due to the partial transmission, while Sample I does not show clear differences from the reference plate. The wave packets corresponding to the A0 mode are extracted to calculate the amplitude spectra by the FFT. The transmission coefficient of the A0 mode is defined analogously to Eq. (3) as

$$
T_{\mathrm{A} 0}(f)=\frac{B_{\mathrm{A} 0}(f)}{A_{\mathrm{A} 0}(f)}
$$

where $A_{\mathrm{A} 0}(f)$ and $B_{\mathrm{A} 0}(f)$ denote the A0 mode amplitudes for the reference and the bonded specimens, respectively. The A0 mode transmission coefficients of four bonded specimens are shown in Fig. 8. It is noted that the A0 mode transmission coefficient of Sample III appears to take a local minimum value close to zero at around $0.56 \mathrm{MHz}$. On the other hand, the transmission coefficients of Samples I and IV do not change significantly with the frequency.

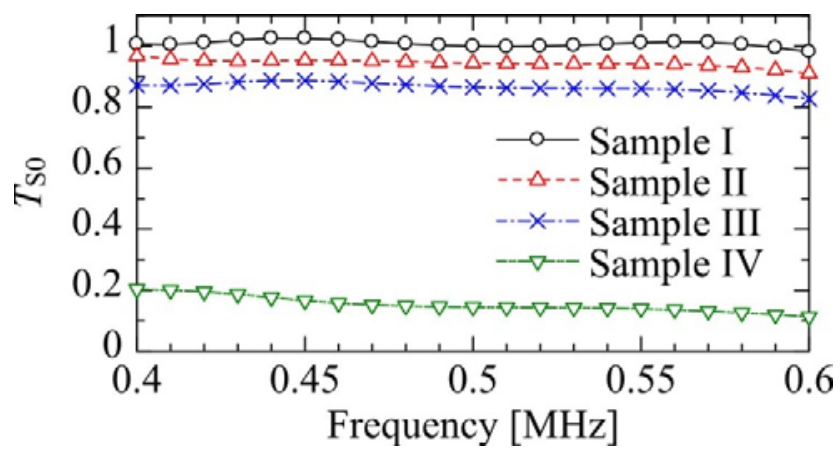

Fig. 6. Frequency dependence of the S0 mode transmission coefficients of Samples 
(a)

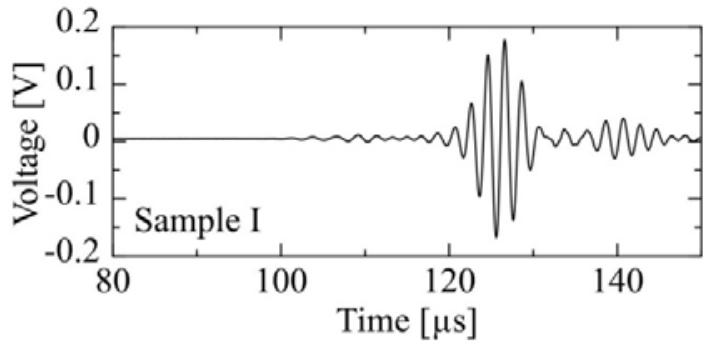

(b)

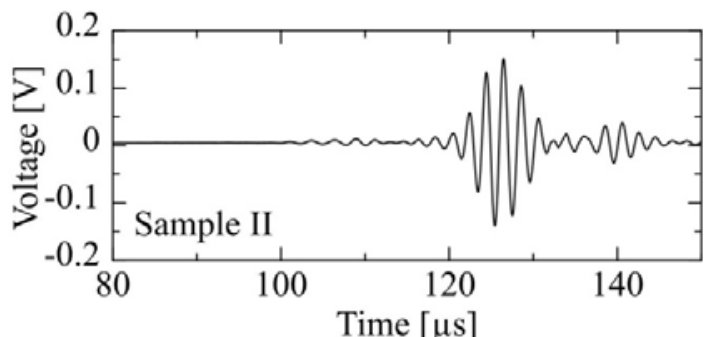

(c)

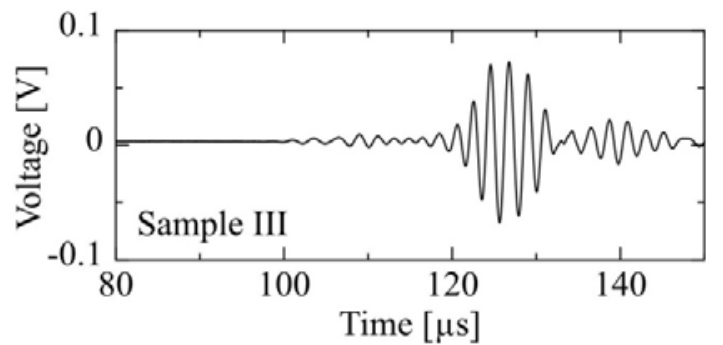

(d)

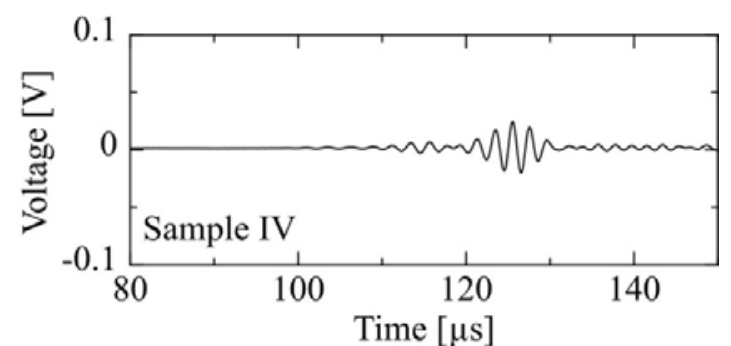

Fig. 7. Measured waveforms of Samples (a) I, (b) II, (c) III, and (d) IV for the A0 mode incidence $(\theta=60 \mathrm{deg})$.

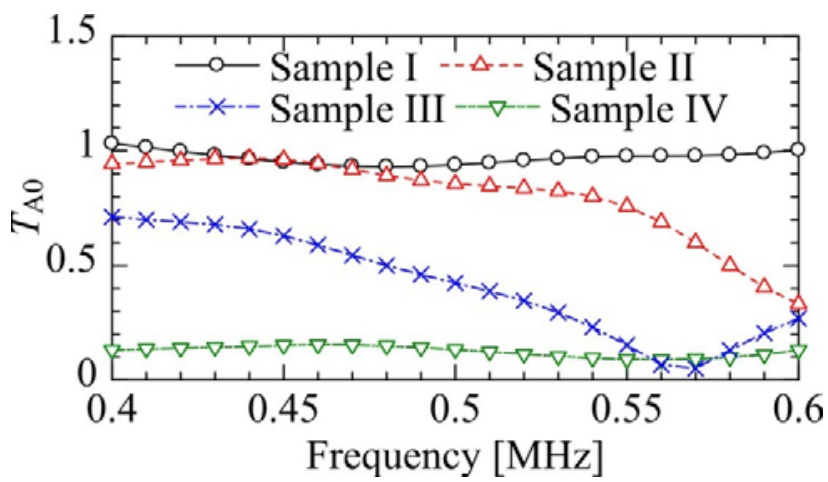

Fig. 8. Frequency dependence of the A0 mode transmission coefficients of Samples I-IV. 


\subsection{Resonance of an adhesive joint subjected to the SO mode incidence}

For the S0 mode incidence at the center frequency $f_{0}=1 \mathrm{MHz}$, Fig. 9 (a) shows the waveform measured in the reference plate. The main wave packet arriving at approximately $150 \mu \mathrm{s}$ is judged to be the S0 mode on the basis of its group velocity $(1.8 \mathrm{~km} / \mathrm{s}$ at $1 \mathrm{MHz})$. The transmitted waveforms of Samples I-IV, shown in Figs. 9 (b)-(e), respectively, are compared to the reference waveform to investigate the effect of the S0 mode interaction with the joint. The shapes of the wave packets of Samples I and II are similar to that of the reference plate, while those of Samples III and IV show long oscillation tails. In Fig. 9 (f), these tail signals are compared to that of the reference waveform for the duration of 160 to $190 \mu$ s.

In order to analyze the frequency content of these tail signals, the FFT is performed for the waveforms using a Hanning window for $165 \mu$ s to $190 \mu \mathrm{s}$. The amplitude spectra of the tail signals for the reference plate and four bonded specimens are shown in Fig. 10 with the vertical axis normalized by the maximum value of the reference spectrum. Samples I and II have the amplitude

(a)

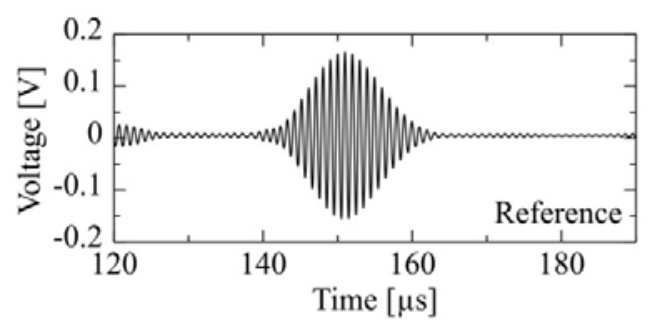

(c)

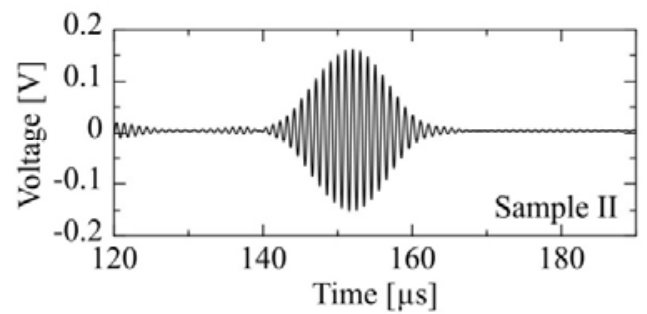

(e)

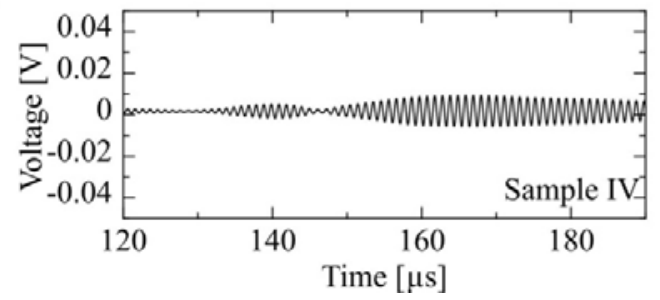

(b)

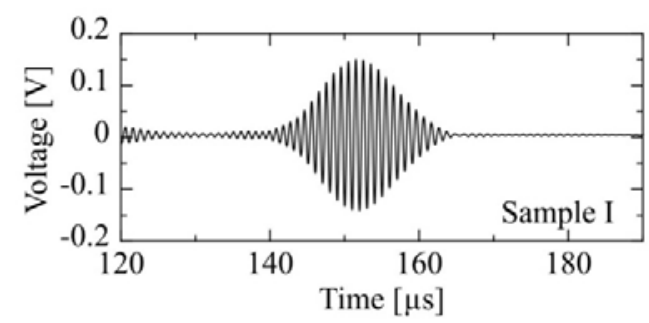

(d)

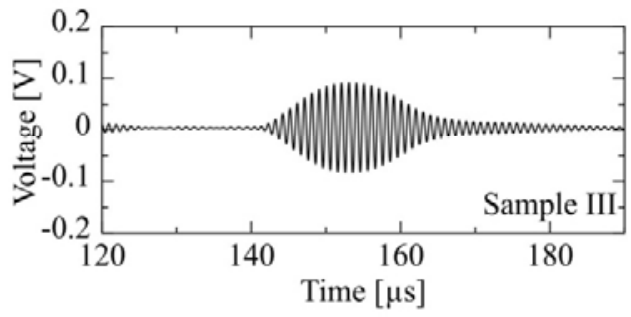

(f)

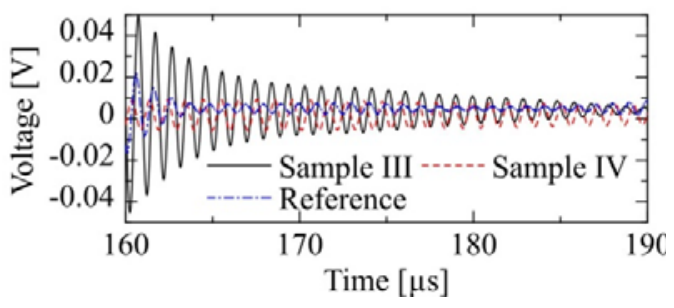

Fig. 9. Measured waveforms of (a) the reference plate, Samples (b) I, (c) II, (d) III, and (e) IV when $\theta=45 \mathrm{deg}$, and (f) comparison of Samples III and IV to the reference plate from $160 \mu$ s to $190 \mu \mathrm{s}$. The center frequency of the input waveform is $1 \mathrm{MHz}$. 


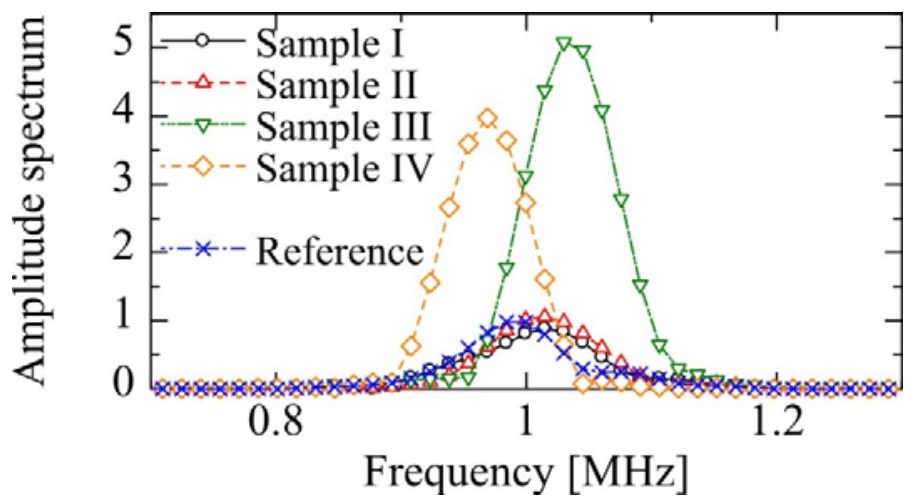

Fig. 10. Amplitude spectra of the measured waveforms from $165 \mu$ s to $190 \mu \mathrm{s}$. The vertical axis is normalized by the maximum value of the reference spectrum.

spectra with magnitude similar to the spectrum of the reference plate. For Samples III and IV, however, the amplitude spectra have significantly increased peaks. The peak frequency appears to shift slightly from the spectral peak of the reference plate $(1 \mathrm{MHz})$, i.e., $1.04 \mathrm{MHz}$ in Sample III and $0.97 \mathrm{MHz}$ in Sample IV. The increased peak amplitudes seen in Fig. 10 indicate that these peak frequencies are the resonance frequencies of the joint [28].

\section{Discussions}

\subsection{Transmission coefficients}

\subsubsection{Application of the spring-type interface model}

In Ref. [12], an imperfect joint of two isotropic elastic plates (density $\rho$, longitudinal and transverse wave velocities $c_{\mathrm{L}}$ and $c_{\mathrm{T}}$, and thickness $d$ ) was modeled as a linear spring-type interface characterized by normal stiffness $K_{\mathrm{N}}$ and tangential stiffness $K_{\mathrm{T}}$. For the incidence of the S0 mode in the frequency range $f d / c_{\mathrm{T}}<1 / 2$, the numerical analysis showed that the amplitude transmission coefficient of the S0 mode decreases monotonically with the frequency. The analysis also showed that the S0 mode transmission coefficient in this frequency range is accurately expressed by the thin-plate theory of extensional wave as 


$$
T_{\mathrm{E}}\left(f, K_{\mathrm{N}}\right)=\frac{\frac{2 K_{\mathrm{N}}}{\rho c_{1} \omega}}{\sqrt{1+4\left(\frac{K_{\mathrm{N}}}{\rho c_{1} \omega}\right)^{2}}},
$$

where $c_{1}=2 c_{\mathrm{T}} \sqrt{1-\left(c_{\mathrm{T}} / c_{\mathrm{L}}\right)^{2}}$ is the velocity of the extensional wave in the plate, and $\omega=2 \pi f$ is the angular frequency.

The above expression based on the thin-plate theory is fitted to the experimental results in Fig. 6. Namely, the value of the normal stiffness $K_{\mathrm{N}}$ which minimizes the evaluation function

$$
J_{\mathrm{E}}\left(K_{\mathrm{N}}\right)=\frac{1}{M} \sum_{n=1}^{M}\left[\frac{T_{\mathrm{E}}\left(f_{n}, K_{\mathrm{N}}\right)-T_{\mathrm{S} 0}^{\exp }\left(f_{n}\right)}{T_{\mathrm{S} 0}^{\exp }\left(f_{n}\right)}\right]^{2},
$$

is sought for, where $f_{n}\left(n=1,2, \ldots, M ; 0.4 \mathrm{MHz}<f_{1}<f_{2}<\ldots<f_{M}<0.6 \mathrm{MHz}\right)$ are the frequencies at which the transmission coefficients $T_{\mathrm{S} 0}^{\exp }$ are obtained from the experiment. The measured transmission coefficients of the S0 mode as well as the theoretical results are shown in Fig. 11 (a). Table 2 gives the estimated value of the normal stiffness $K_{\mathrm{N}}$ for each bonded plate specimen. The stiffness of Sample I was difficult to quantify since the evaluation function in Eq. (6) was monotonically decreasing in the range $0.1 \mathrm{GPa} / \mathrm{mm}<K_{\mathrm{N}}<1000 \mathrm{GPa} / \mathrm{mm}$ : namely, Sample I was indistinguishable from the reference plate $\left(K_{\mathrm{N}}=\infty\right)$. Therefore, among Samples I, II, and III bonded with the same compression time, the higher interfacial stiffness is associated with the specimen which was bonded with higher pressure and has smaller adhesive thickness. Sample IV, bonded with low pressure and short compression time, has significantly small interfacial stiffness compared to the others.

In Ref. [12], it is also shown that the transmission coefficient for the A0 mode incidence exhibits non-monotonic dependence on the frequency, and has a zero at a certain frequency which depends on the interfacial stiffnesses $K_{\mathrm{N}}$ and $K_{\mathrm{T}}$. Mindlin's thin-plate theory for flexural waves [30] can reasonably reproduce the numerical results of the A0 mode reflection and transmission at the imperfect joint. In the present study, the transmission coefficient of the flexural wave is obtained in the same manner as Ref. [12], except that the shear factor introduced in Mindlin's theory is set as $\kappa=\pi^{2} / 12$ to equalize the cut-off frequencies of the higher-order flexural mode $\left(f d / c_{\mathrm{T}}=\sqrt{3 \kappa} / \pi\right)$ and the A1 Lamb mode $\left(f d / c_{\mathrm{T}}=1 / 2\right)$. Unlike Eq. (5), the transmission coefficient is a function of both interfacial stiffnesses $K_{\mathrm{N}}$ and $K_{\mathrm{T}}$. Then, the normal stiffness $K_{\mathrm{N}}$ is 
fixed to the value determined above from the S0 mode transmission coefficient. The theoretical relation for the thin-plate theory of flexural wave is fitted to the measured transmission coefficients of the A0 mode $T_{\mathrm{A} 0}^{\mathrm{exp}}$ by minimizing the following function with respect to the interfacial tangential stiffness $K_{\mathrm{T}}$

$$
J_{\mathrm{F}}\left(K_{\mathrm{N}}, K_{\mathrm{T}}\right)=\frac{1}{M} \sum_{n=1}^{M}\left[\frac{T_{\mathrm{F}}\left(f_{n}, K_{\mathrm{N}}, K_{\mathrm{T}}\right)-T_{\mathrm{A} 0}^{\exp }\left(f_{n}\right)}{T_{\mathrm{A} 0}^{\exp }\left(f_{n}\right)}\right]^{2},
$$

Table 2 Interfacial stiffnesses estimated by the transmission measurement of Lamb waves based on the spring-type interface model.

\begin{tabular}{c|ccc}
\hline \hline & $K_{\mathrm{N}}[\mathrm{GPa} / \mathrm{mm}]$ & $K_{\mathrm{T}}[\mathrm{GPa} / \mathrm{mm}]$ & $K_{\mathrm{T}} / K_{\mathrm{N}}$ \\
\hline Sample II & 67 & 21 & 0.32 \\
Sample III & 41 & 12 & 0.29 \\
Sample IV & 3.5 & 0.24 & 0.07 \\
\hline \hline
\end{tabular}

(a)

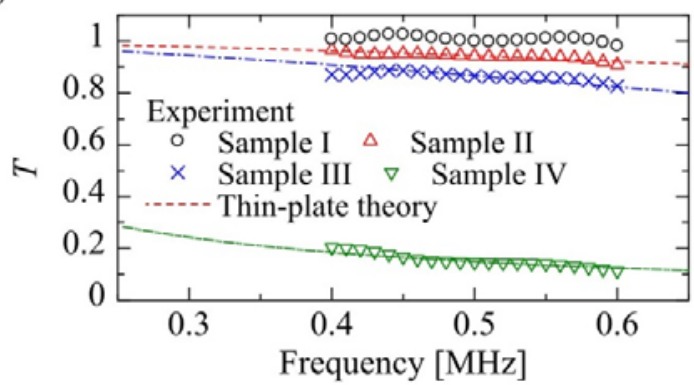

(c)

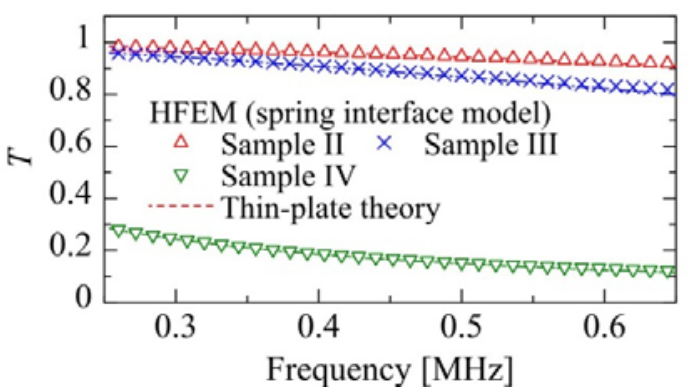

(b)

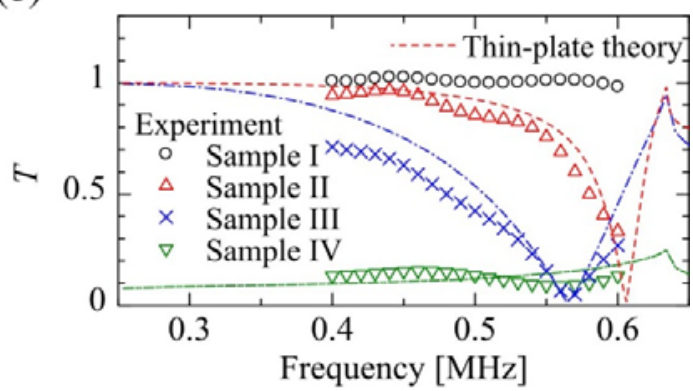

(d)

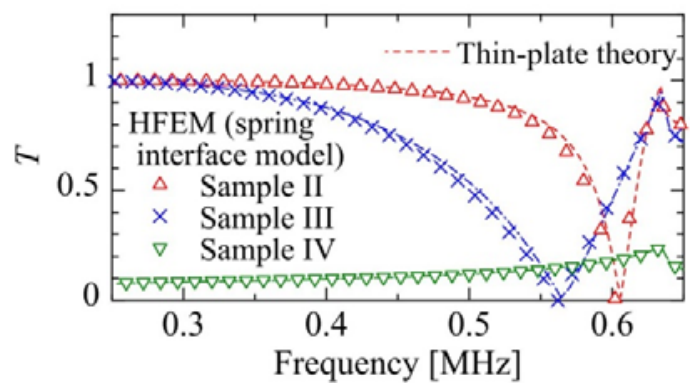

Fig. 11. Comparison of the measured transmission coefficients of the (a) S0 and (b) A0 modes to the thin-plate theory with the spring-type interface model, and comparison of the thin-plate theory to the HFEM for the transmission coefficients of the (c) S0 and (d) A0 modes. 
where $T_{\mathrm{F}}\left(f, K_{\mathrm{N}}, K_{\mathrm{T}}\right)$ is the amplitude transmission coefficient of the lower-order flexural mode in Mindlin's theory. The measured transmission coefficients of the A0 mode as well as the theoretical results are shown in Fig. 11 (b). Table 2 provides the estimated values of the tangential stiffness $K_{\mathrm{T}}$ and stiffness ratio $K_{\mathrm{T}} / K_{\mathrm{N}}$. It is seen that the difference of the bonding procedure gives influence on the resulting tangential stiffness in a similar manner to the normal stiffness. Therefore it is suggested that the bonding quality of the joint can be evaluated by the S0 and A0 mode transmission characteristics. It is noted that the theoretical transmission coefficients have sharp peaks at $0.63 \mathrm{MHz}$ which corresponds to the cut-off frequency of the A1 mode: this frequency is out of the range of the present measurement so these peaks are not confirmed experimentally.

In Figs. 11 (c) and (d), the transmission coefficients of the S0 and A0 modes, obtained by the two-dimensional elastodynamic analysis using the hybrid finite element method (HFEM) [12, 23 ] and the so-identified interfacial stiffnesses, are compared to the corresponding results of the thin-plate theories. The results obtained by the thin-plate theories and the HFEM are in good agreement, indicating that the interfacial stiffnesses identified above remain valid as far as the adhesive joint is modeled as a spring-type interface.

\subsubsection{Comments on the spring-type interface model}

The spring-type interface model is known to be an adequate description of adhesively bonded interfaces when the thickness of adhesive is sufficiently small compared to the wavelength $[14,15]$. In order to check the validity of the spring-type interface model for the specimens used here, the theoretical results based on the spring-type interface model are compared to those when the adhesive is modeled as an elastic strip of finite length $h_{\mathrm{A}}$. In this adhesive zone model, it is first assumed that the adhesive is perfectly bonded to the plates. The velocities of longitudinal and transverse waves in the adhesive, denoted by $c_{\mathrm{LA}}$ and $c_{\mathrm{TA}}$, respectively, are then estimated from the simple relations [15]

$$
K_{\mathrm{N}}=\frac{\rho_{\mathrm{A}} c_{\mathrm{LA}}^{2}}{h_{\mathrm{A}}}, K_{\mathrm{T}}=\frac{\rho_{\mathrm{A}} c_{\mathrm{TA}}^{2}}{h_{\mathrm{A}}},
$$

where the parameter $\rho_{\mathrm{A}}$ denotes the mass density of the adhesive. To obtain specific numerical results, the mass density of the adhesive is set as $\rho_{\mathrm{A}}=1230 \mathrm{~kg} / \mathrm{m}^{3}$ (the value for epoxy [31] is used here since the correct density of cyanoacrylate-based adhesive is unavailable). The bulk 
wave velocities $c_{\mathrm{LA}}$ and $c_{\mathrm{TA}}$ calculated by Eq. (8) are shown in Table 3 for Samples II-IV. The velocities for Samples II and III appear reasonable for polymer-based solids. For Sample IV, the result shows remarkably small wave velocities which appear unreasonable for the same adhesive used in Samples I-III. Since Sample IV was manufactured with low bonding pressure and short compression time, it is likely that the interfaces between the adhesive and the plates are poorly bonded. To examine this possibility, the results of Sample IV are discussed separately on the basis of a different model in the later section.

For Samples II and III, the transmission coefficients of the S0 and A0 Lamb modes across the adhesive zone are calculated by the HFEM. Figure 12 shows the two-dimensional model of semi-infinite isotropic elastic plates $|z|<d / 2(d=2.5 \mathrm{~mm})$ with an adhesive zone $|x|<h_{\mathrm{A}} / 2$. The plates are made of aluminum $\left(c_{\mathrm{L}}=6.4 \mathrm{~km} / \mathrm{s}, c_{\mathrm{T}}=3.17 \mathrm{~km} / \mathrm{s}\right.$, and $\left.\rho=2700 \mathrm{~km} / \mathrm{m}^{3}\right)$ and under the plane-strain condition in the $x$ - $z$ coordinates. The bounded area $|x|<h_{\mathrm{A}} / 2+0.24 d$ is discretized by four-node isoparametric elements. The length of the elements in the $z$-direction is set as $\Delta z=$ $d / 25$, while the length in the $x$-direction is $\Delta x=h_{\mathrm{A}} / 4$ in the adhesive zone $\left(|x|<h_{\mathrm{A}} / 2\right)$ and $\Delta x=$ $d / 25$ in the plate region $\left(h_{\mathrm{A}} / 2<|x|<h_{\mathrm{A}} / 2+0.24 d\right)$, respectively. The displacement fields outside the discretized area are expressed as the series of six symmetric and six antisymmetric Lamb

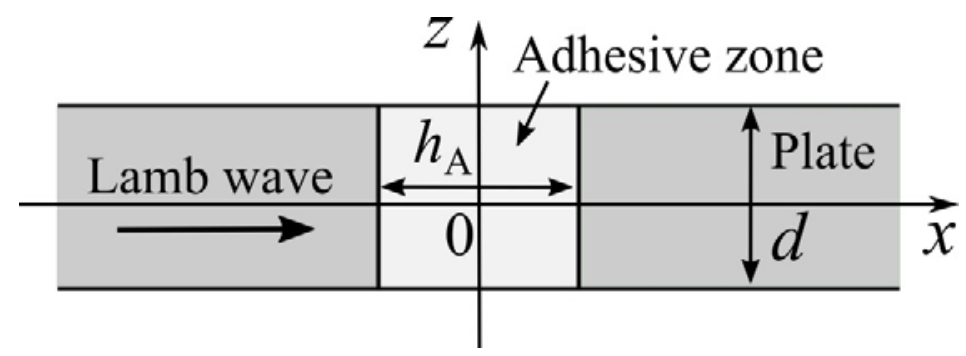

Fig. 12. Adhesive zone model of bonded aluminum plates.

Table 3 Wave velocities of adhesive estimated from the interfacial stiffnesses.

\begin{tabular}{c:cc}
\hline \hline & $c_{\mathrm{LA}}[\mathrm{km} / \mathrm{s}]$ & $c_{\mathrm{TA}}[\mathrm{km} / \mathrm{s}]$ \\
\hline Sample II & 2.66 & 1.51 \\
Sample III & 2.99 & 1.60 \\
Sample IV & 0.672 & 0.175 \\
\hline \hline
\end{tabular}


modes. Continuity condition is applied to the displacement and stress components at $x= \pm h_{\mathrm{A}} / 2$. The number of total nodes and elements is 442 and 400, respectively.

The calculated transmission coefficients of the S0 and A0 Lamb modes of the adhesive zone model are shown in Figs. 13 (a)-(d). In Fig. 13, two kinds of numerical results are shown. The first result is referred to as the adhesive zone model (A), for which the wave velocities $c_{\mathrm{LA}}$ and $c_{\mathrm{TA}}$ in the adhesive are determined from Eq. (8) as mentioned above using the interfacial stiffnesses identified experimentally based on the application of the spring-type interface model ( $c_{\mathrm{LA}}$ and $c_{\mathrm{TA}}$ are shown in Table 3 ). In the second result, referred to as the adhesive zone model (B), $c_{\mathrm{LA}}$ and $c_{\mathrm{TA}}$ are chosen as in Table 4 so that the numerical results of the adhesive zone model

(a)

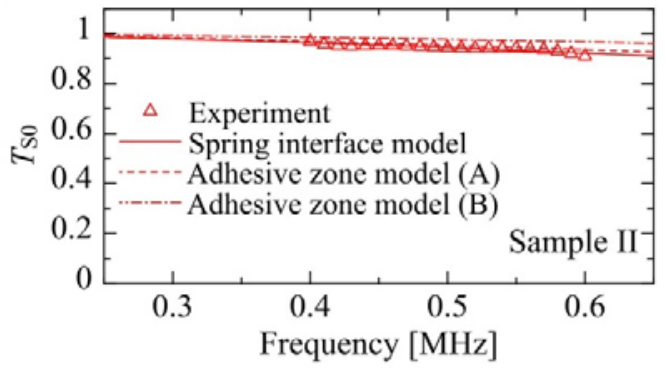

(c)

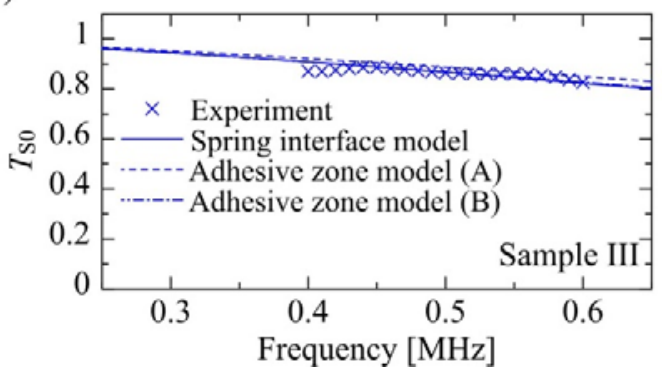

(b)

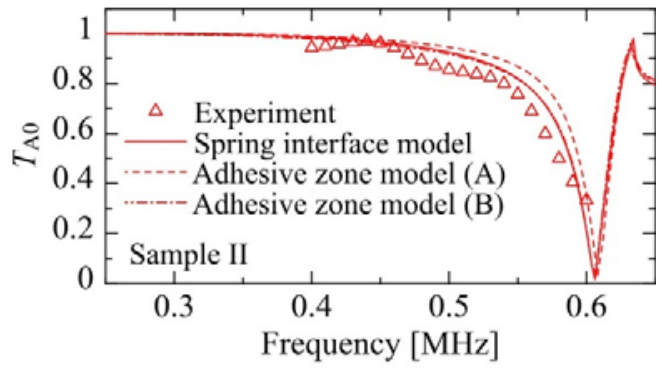

(d)

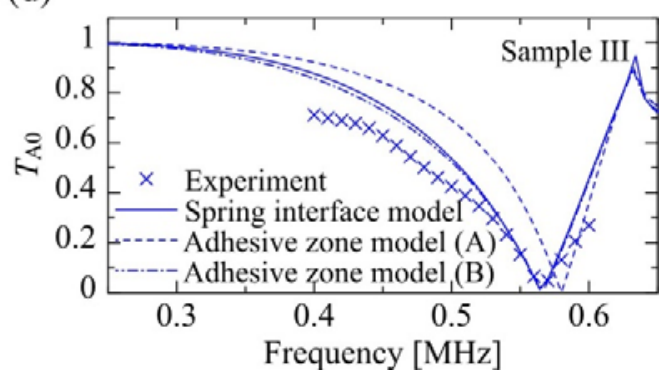

Fig. 13. Comparison of the theoretical transmission coefficients for the spring-type interface model, and the adhesive zone model (A) and (B) to the experimental results: (a) S0 and (b) A0 modes for Sample II, and (c) S0 and (d) A0 modes for Sample III.

Table 4 Wave velocities of adhesive used for the adhesive zone model (B).

\begin{tabular}{c|cc}
\hline \hline & $c_{\mathrm{LA}}[\mathrm{km} / \mathrm{s}]$ & $c_{\mathrm{TA}}[\mathrm{km} / \mathrm{s}]$ \\
\hline Sample II & 3.26 & 1.48 \\
Sample III & 2.89 & 1.47 \\
\hline
\end{tabular}


fit best to the measured transmission coefficients. It is shown in Figs. 13 (a) and (c) that the spring-type interface model and the two results (A) and (B) of the adhesive zone model give almost identical curves for the S0 mode. Namely, the spring-type interface model can reproduce the transmission coefficient of the S0 mode accurately. In Figs. 13 (b) and (d) for the A0 mode, the three curves of the numerical analysis are in qualitative agreement regarding the frequency dependence of the transmission coefficient and the local minimum behavior. A quantitative deviation can be seen in Figs. 13 (b) and (d) for the adhesive zone model (A) from the other two. This is due to the non-negligible thickness of adhesives and the fact that $c_{\mathrm{LA}}$ and $c_{\mathrm{TA}}$ used in the adhesive zone model (A) are fixed as the values determined from Eq. (8). When these parameters are chosen as in Table 4, the adhesive zone model (B) can reproduce the experimental results as well as the spring-type interface model. As a whole, the use of the spring-type interface model is justifiable to qualitatively interpret the transmission coefficients for Samples II and III in Figs. 11 (a) and (b).

\subsubsection{Application of the double spring-type interface model}

The assumption of perfect adhesive-plate bonding in the adhesive zone model gave unreasonably small bulk wave velocities of adhesive for Sample IV (Table 3), suggesting the possibility that the adhesive-plate interfaces are poorly bonded. To take into account this effect, the adhesive is modeled as an elastic strip bonded to the plates by spring-type interfaces at $x=$ $\pm h_{\mathrm{A}} / 2$. A similar approach has been used by Castaings [32] to interpret the shear horizontal wave propagation in an adhesively bonded metallic plate, also incorporating the viscoelastic nature of the adhesive. In the present discussion, the normal and tangential stiffnesses $\left(S_{\mathrm{N}}, S_{\mathrm{T}}\right)$ are assumed to be the same at the left and right interfaces $x= \pm h_{\mathrm{A}} / 2$. The thickness of the adhesive is set as $h_{\mathrm{A}}$ $=0.16 \mathrm{~mm}$, according to the measured value shown in Table 1 . The bulk wave velocities of the adhesive are assumed to be $c_{\mathrm{LA}}=2.66 \mathrm{~km} / \mathrm{s}$ and $c_{\mathrm{TA}}=1.51 \mathrm{~km} / \mathrm{s}$, in accordance with the values of Sample II given in Table 3. The interfacial stiffnesses which minimize the following function

$$
J_{\mathrm{L}}\left(S_{\mathrm{N}}, S_{\mathrm{T}}\right)=J_{\mathrm{S}}\left(S_{\mathrm{N}}, S_{\mathrm{T}}\right)+J_{\mathrm{A}}\left(S_{\mathrm{N}}, S_{\mathrm{T}}\right) \text {, }
$$

are identified by using the Nelder-Mead method [33], where

$$
J_{\mathrm{S}}\left(S_{\mathrm{N}}, S_{\mathrm{T}}\right)=\frac{1}{M} \sum_{n=1}^{M}\left[\frac{T_{\mathrm{S} 0}^{\text {theo }}\left(f_{n}, S_{\mathrm{N}}, S_{\mathrm{T}}\right)-T_{\mathrm{S} 0}^{\exp }\left(f_{n}\right)}{T_{\mathrm{S} 0}^{\exp }\left(f_{n}\right)}\right]^{2},
$$




$$
J_{\mathrm{A}}\left(S_{\mathrm{N}}, S_{\mathrm{T}}\right)=\frac{1}{M} \sum_{n=1}^{M}\left[\frac{T_{\mathrm{A} 0}^{\text {theo }}\left(f_{n}, S_{\mathrm{N}}, S_{\mathrm{T}}\right)-T_{\mathrm{A} 0}^{\exp }\left(f_{n}\right)}{T_{\mathrm{A} 0}^{\exp }\left(f_{n}\right)}\right]^{2},
$$

and $T_{\mathrm{S} 0}^{\text {theo }}$ and $T_{\mathrm{A} 0}^{\text {theo }}$ denote the transmission coefficients of the S0 and A0 modes calculated by the HFEM, respectively. As a result, the interfacial stiffnesses are estimated as $S_{\mathrm{N}}=5.6$ $\mathrm{GPa} / \mathrm{mm}$ and $S_{\mathrm{T}}=3.2 \mathrm{GPa} / \mathrm{mm}\left(S_{\mathrm{T}} / S_{\mathrm{N}}=0.57\right)$. The theoretical transmission coefficients of Sample IV based on this model are compared to the experimental results in Figs. 14 (a) and (b). As shown in Fig. 14, the double spring-type interface model reproduces low transmission coefficients of both S0 and A0 modes observed in the experiment as well as the spring-type interface model. It is noted that the agreement between the double spring-type interface model and the measurements can be better if $c_{\mathrm{LA}}$ and $c_{\mathrm{TA}}$ are also optimized, but this is out of the scope of this analysis. The analysis shown here indicates that the small interfacial stiffnesses of Sample IV based on the single spring-type interface model reflect poor bonding at the adhesive-plate interfaces.

(a)

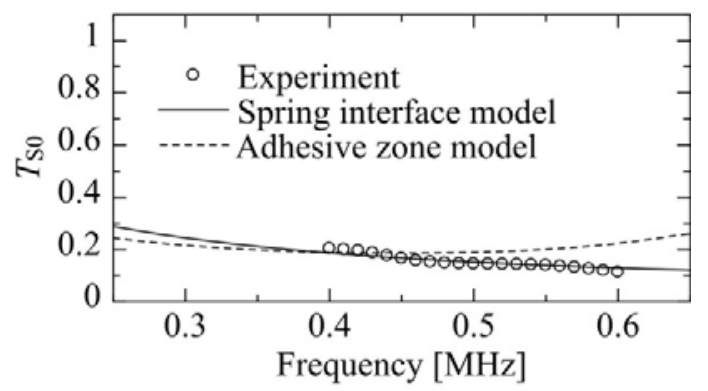

(b)

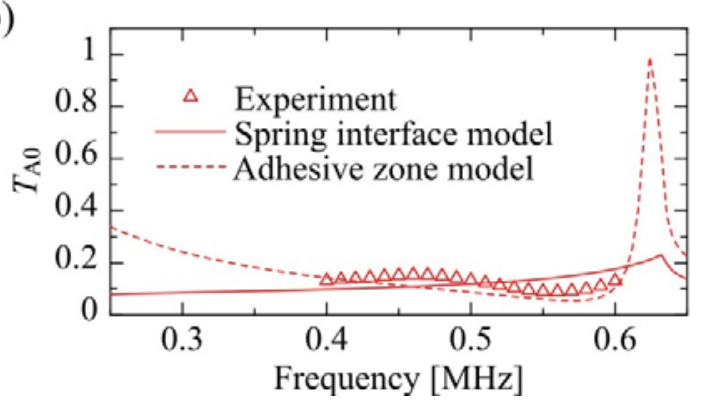

Fig. 14. Comparison of the transmission coefficients of the (a) S0 and (b) A0 modes obtained from the single spring-type interface model and the adhesive zone model with double spring interfaces to the experimental result of Sample IV. 


\subsection{Resonance frequencies}

The numerical analysis in Ref. [23] has shown that when an imperfect joint of plates is modeled as a spring-type interface, two resonance frequencies of the joint exist in the frequency range $f_{\mathrm{FR}}<f<f_{\mathrm{ZGV}}$, where $f_{\mathrm{FR}}$ is the resonance frequency of a free edge and $f_{\mathrm{ZGV}}$ is the zero group velocity frequency of the $\mathrm{S} 1 \mathrm{Lamb}$ mode $[34,35]$. The analysis has also shown that the resonance frequencies increase with the interfacial stiffnesses to approach $f_{\mathrm{ZGV}}$ asymptotically. The above range corresponds to $0.95 \mathrm{MHz}<f<1.16 \mathrm{MHz}$ for aluminum plates of thickness $2.5 \mathrm{~mm}$, which is covered by the present measurement as shown in Fig. 10. Predoi and Rousseau [10] analyzed the interaction of Lamb waves with an elastic strip of finite thickness bonded between semi-infinite elastic plates, and found a similar type of resonance at the frequency close to the free-edge resonance. They also show the occurrence of another resonance phenomenon, the strip resonance, when the wavelength meets certain relations with the strip thickness. In Ref. [10], relatively thick strip was considered as compared to the plate thickness. The frequency for the strip resonance increases as the strip thickness decreases. For the adhesive bonding considered in this study, the adhesive thicknesses (Table 1) are relatively small so that the smallest frequencies expected for the strip resonance, i.e., the adhesive thickness being equal to the quarter wavelength of $\mathrm{S} 0$ or $\mathrm{A} 0$ mode, are higher than the frequency range of the present measurement. Therefore, the strip resonance is not considered in the present discussion.

Using the interfacial stiffnesses in Table 2, the numerical analysis based on the spring-type interface model gives the theoretical resonance frequencies of Samples II-IV as shown in Table 5. For Samples II and III, the numerical analysis can identify only one resonance frequency. In Ref. [23], it has been shown that in certain ranges of interfacial stiffnesses the two resonance

Table 5 Experimental and theoretical values of the resonance frequencies.

\begin{tabular}{c|ccc}
\hline \hline & Experiment & \multicolumn{2}{|c}{ Theory [MHz] } \\
\cline { 3 - 4 } & {$[\mathrm{MHz}]$} & Spring interface & Adhesive zone \\
\hline Sample II & N/A & 1.10 & $1.08,1.10^{\mathrm{a}}$ \\
Sample III & 1.04 & 1.07 & $1.00,1.05^{\mathrm{a}}$ \\
Sample IV & 0.97 & $0.96,0.97$ & $1.00,1.01^{\mathrm{b}}$ \\
\hline \hline
\end{tabular}

${ }^{a}$ Adhesive-plate interfaces are assumed to be perfectly bonded.

${ }^{\mathrm{b}}$ Adhesive-plate interfaces are assumed to be spring-type interfaces. 
frequencies approach each other so closely and appear as a single resonance.

The theoretical resonance frequencies for Samples II and III are also obtained using the adhesive zone model based on the out-of-plane displacement amplitude at $(x, z)=\left(h_{\mathrm{A}} / 2, d / 2\right)$ for $0.9 \mathrm{MHz}<f<1.2 \mathrm{MHz}$ by the HFEM. For Samples II and III, the perfect bonding is assumed for the adhesive-plate interfaces and the bulk wave velocities given in Table 3 are used. Table 5 gives the resonance frequencies obtained by the analysis. If the bulk wave velocities in Table 4 are used in the analysis, the adhesive zone model predicts two resonance frequencies as $0.99 \mathrm{MHz}$ and 1.03 $\mathrm{MHz}$, which are close to the results in Table 5.

Experimentally, Sample II did not show measurable resonance, and Sample III gave only a single resonance frequency at $1.04 \mathrm{MHz}$. Namely, the present measurement was not able to confirm the existence of two resonance frequencies at the adhesive joint. This is, however, not contradictory to the theory. It should be noted that the theoretically predicted resonance frequencies are very close to each other in all cases, and the measurement has limited frequency resolution when the resonance frequencies are to be obtained from the spectral analysis of the transient waveform of finite length. The measured resonance frequency of $1.04 \mathrm{MHz}$ of Sample III is reasonably close to the theoretical results shown in Table 5.

Resonance is also expected to occur analogously if the effect of imperfect interfaces is taken into account between the plates and adhesive. By using the adhesive zone model with double spring-type interfaces, the out-of-plane displacement amplitude at the plate edge $(x, z)=$ $\left(h_{\mathrm{A}} / 2, d / 2\right)$ is calculated for $0.9 \mathrm{MHz}<f<1.2 \mathrm{MHz}$ by the HFEM for Sample IV. As a result, the displacement amplitude takes local maxima at the frequencies given in Table $5(1.00 \mathrm{MHz}$ and $1.01 \mathrm{MHz}$ ). If the effect of the imperfect interfaces is neglected and perfect bonding is assumed between the plates and adhesive, the resonance frequencies are obtained as $1.06 \mathrm{MHz}$ and 1.09 $\mathrm{MHz}$ by the HFEM. Therefore the presence of the spring interfaces gives smaller resonance frequencies of the adhesive joint. The measured resonance frequency $(0.97 \mathrm{MHz})$ is reasonably close to the frequencies which are predicted on the basis of the single spring-type interface model $(0.96 \mathrm{MHz}$ and $0.97 \mathrm{MHz})$ as well as the double spring-type interface model $(1.00 \mathrm{MHz}$ and $1.01 \mathrm{MHz}$ ). It is noted here again that the results of the double spring-type interface model may improve if the adhesive parameters are chosen to best fit the measurement.

In the present experiment, the resonance frequencies have been identified for Samples III and IV, while no resonance effect has been confirmed for Samples I and II. Namely, the resonant 
behavior has been observed in the transmitted waveform for weakly bonded joints. The resonance frequencies experimentally obtained for Samples III and IV are fairly close to those predicted theoretically. The resonance frequency found for Sample IV $(0.97 \mathrm{MHz})$ is close to the free-edge resonance frequency $(0.95 \mathrm{MHz})$. This result can be attributed to the poor bonding of the adhesive-plate interfaces in Sample IV.

\section{Conclusion}

In this study, the interaction of Lamb waves with an adhesive butt joint of aluminum alloy plates has been investigated experimentally. Namely, the transmission characteristics of the lowest-order symmetric (S0) and antisymmetric (A0) Lamb modes and the resonance at the joint have been evaluated when subjected to the S0 mode incidence. Adhesively bonded plate specimens with different joint properties were prepared to examine the effect of the bonding condition on the Lamb wave characteristics. It is shown that the transmission coefficient for the S0 mode incidence shows only weak frequency dependence, while that for the A0 mode incidence exhibits distinct frequency dependence for different specimens. The spring-type interface model has been applied to interpret the experimental data of the transmission coefficients, and the interfacial stiffnesses have been identified for each specimen. The identified interfacial stiffnesses have been shown to be correlated with the bonding condition of the adhesive. Particularly low interfacial stiffnesses identified for the poorly bonded specimen have been attributed to the imperfect nature of the adhesive-plate interfaces. Furthermore, the resonance frequencies of the joint have been identified for weakly bonded specimens by the long oscillation in the transmitted waveform of the S0 mode. The theoretical models used to discuss the transmission coefficients have been shown to give reasonable estimates of the resonance frequencies.

\section{Acknowledgements}

This work has been supported by JSPS KAKENHI Grant Numbers 26-2190 and 25289005.

\section{References}

[1] Y. Cho, D. D. Hongerholt, J. L. Rose, Lamb wave scattering analysis for reflector characterization, IEEE Trans. Ultrason. Ferroelectr. Freq. Control 44 (1997) 44-52. 
[2] M. Castaings, E. Le Clezio, B. Hosten, Modal decomposition method for modeling the interaction of Lamb waves with cracks, J. Acoust. Soc. Am. 112 (2002) 2567-2582.

[3] M. J. S. Lowe, O. Diligent, Low-frequency reflection characteristics of the $s_{0}$ Lamb wave from a rectangular notch in a plate, J. Acoust. Soc. Am. 111 (2002) 64-74.

[4] A. Gunawan and S. Hirose, Mode-exciting method for Lamb wave-scattering analysis, $J$. Acoust. Soc. Am. 115 (2004) 996-1005.

[5] P. B. Nagy and L. Adler, Nondestructive evaluation of adhesive layer of adhesive joints by guided waves, J. Appl. Phys. 66 (1989) 4658.

[6] S. I. Rokhlin, Lamb wave interaction with lap-shear adhesive joints: Theory and experiment, $J$. Acoust. Soc. Am. 89 (1991) 2758-2765.

[7] M. J. S. Lowe, R. E. Challis, C. W. Chan, The transmission of Lamb waves across adhesively bonded lap joints, J. Acoust. Soc. Am. 107 (2000) 1333-1345.

[8] K. Heller, L. J. Jacobs, J. Qu, Characterization of adhesive bond properties using Lamb waves, NDT\&E Int. 33 (2000) 555-563.

[9] F. Lanza di Scalea, P. Rizzo, A. Marzani, Propagation of ultrasonic guided waves in lap-shear adhesive joints: Case of incident $a_{0}$ Lamb wave, J. Acoust. Soc. Am. 115 (2004) 146-156.

[10] M. V. Predoi, M. Rousseau, Lamb waves propagation in elastic plane layers with a joint strip, Ultrasonics 43 (2005) 551-559.

[11] P. Puthillath, J. M. Galan, B. Ren, C. J. Lissenden, J. L. Rose, Ultrasonic guided wave propagation across waveguide transitions: Energy transfer and mode conversion, J. Acoust. Soc. Am. 133 (2013) 2624-2633.

[12] N. Mori, S. Biwa, T. Hayashi, Reflection and transmission of Lamb waves at an imperfect joint of plates, J. Appl. Phys. 113 (2013) 074901.

[13] H. G. Tattersall, The ultrasonic pulse-echo technique as applied to adhesion testing, J. Phys. D: Appl. Phys. 6 (1973) 819-832.

[14] J.-M. Baik, R. B. Thompson, Ultrasonic scattering from imperfect interfaces: A quasi-static model, J. Nondestruct. Eval. 4 (1984) 177-196.

[15] A. Pilarski, J. L. Rose, Ultrasonic oblique incidence for improved sensitivity in interface weakness determination, NDT Int. 21 (1988) 241-246.

[16] B. W. Drinkwater, R. S. Dwyer-Joyce, P. Cawley, A study of the interaction between ultrasound and a partially contacting solid-solid interface, Proc. R. Soc. London, Ser. A 452 (1996) 2613-2628.

[17] C. Pecorari, Scattering of a Rayleigh wave by a surface-breaking crack with faces in partial contact, Wave Motion 33 (2001) 259-270.

[18] B. W. Drinkwater, M. Castaings, B. Hosten, The measurement of $\mathrm{A}_{0}$ and $\mathrm{S}_{0} \mathrm{Lamb}$ wave attenuation to determine the normal and shear stiffnesses of a compressively loaded interface, J. Acoust. Soc. Am. 113 (2003) 3161-3170.

[19] S. Biwa, A. Suzuki, N. Ohno, Evaluation of interface wave velocity, reflection coefficients and interfacial stiffnesses of contacting surfaces, Ultrasonics 43 (2005) 495-502. 
[20] B. Hosten, M. Castaings, Finite elements methods for modeling the guided waves propagation in structures with weak interfaces, J. Acoust. Soc. Am. 117 (2005) 1108-1113.

[21] S. Biwa, S. Hiraiwa, E. Matsumoto, Stiffness evaluation of contacting surfaces by bulk and interfacial waves, Ultrasonics 47 (2007) 123-129.

[22] M. V. Golub, A. Boström, Interface damage modeled by spring boundary conditions for in-plane elastic waves, Wave Motion 48 (2011) 105-115.

[23] N. Mori, S. Biwa, Resonance of an imperfect joint of plates by the lowest-order symmetric Lamb mode, J. Acoust. Soc. Am. 137 (2015) 3139-3148.

[24] J.-B. Ihn, F.-K. Chang, Detection and monitoring of hidden fatigue crack growth using a built-in piezoelectric sensor/actuator network: I. Diagnostics, Smart Mater. Struct. 13 (2004) 609-620.

[25] B. C. Lee, W. J. Staszewski, Modelling of Lamb wave interaction with open and closed fatigue cracks for damage detection, IOP Conf. Ser.: Mater. Sci. Eng. 10 (2010) 012059.

[26] H. Cho, C. J. Lissenden, Structural health monitoring of fatigue crack growth in plate structures with ultrasonic guided waves, Struct. Health Monit. 11 (2011) 393-404.

[27] J. L. Rose, Ultrasonic Waves in Solid Media, Cambridge University Press, 1999.

[28] N. Mori, S. Biwa, Interaction of Lamb waves with an imperfect joint of plates: reflection, transmission and resonance, Phys. Procedia 70 (2015) 480-483.

[29] E. Le Clezio, M. V. Predoi, M. Castaings, B. Hosten, M. Rousseau, Numerical predictions and experiments on the free-plate edge mode, Ultrasonics 41 (2003) 25-40.

[30] R. D. Mindlin, Influence of rotatory inertia and shear on flexural motions of isotropic elastic plates, J. Appl. Mech. 18 (1951) 31-38.

[31] S. Biwa, Y. Watanabe, N. Ohno, Analysis of wave attenuation in unidirectional viscoelastic composites by a differential scheme, Comp. Sci. Tech. 63 (2003) 237-247.

[32] M. Castaings, SH ultrasonic guided waves for the evaluation of interfacial adhesion, Ultrasonics 54 (2014) 1760-1775.

[33] J. A. Nelder, R. Mead, A simplex method for function minimization, Comput. J. 7 (1964) 308-313.

[34] A. Gibson, J. S. Popovics, Lamb wave basis for impact-echo method analysis, J. Eng. Mech. 131 (2005) 438-443.

[35] D. Clorennec, C. Prada, D. Royer, Local and noncontact measurements of bulk acoustic wave velocities in thin isotropic plates and shells using zero group velocity Lamb modes, J. Appl. Phys. 101 (2007) 034908. 